\title{
First results obtained using the CENBG nanobeam line: Performances and applications
}

\author{
Ph. Barberet ${ }^{\mathrm{a}, *}$, L. Daudin ${ }^{\mathrm{a}}$, N. Gordillo ${ }^{\mathrm{a}}$, S. Sorieul ${ }^{\mathrm{a}}$, M. Simon ${ }^{\mathrm{a}}$, H. Seznec ${ }^{\mathrm{a}}$, I. Idarraga ${ }^{\mathrm{b}}$, S. Incerti $^{\mathrm{a}}$, \\ A. Balana ${ }^{\mathrm{a}}$, Ph. Moretto ${ }^{\mathrm{a}}$ \\ a Centre d'Etudes Nucléaires de Bordeaux-Gradignan, Université Bordeaux 1, CNRS/IN2P3, Chemin du Solarium, BP 120, F-33175 Gradignan cedex, France \\ ${ }^{\mathrm{b}}$ Institut de Radioprotection et de Sûreté Nucléaire, IRSN, 13115 St Paul Lez Durance, France
}

Keywords:

Ion nanobeam

uclear microprobe

Magnetic quadrupoles

Ion beam optics

Ion beam analysis

\begin{abstract}
A B S T R A C T
A high resolution focused beam line has been recently installed on the AIFIRA ("Applications Interdisciplinaires des Faisceaux d'Ions en Région Aquitaine") facility at CENBG. This nanobeam line, based on a doublet-triplet configuration of Oxford Microbeam Ltd. OM-50 $0^{\mathrm{TM}}$ quadrupoles, offers the opportunity to focus protons, deuterons and alpha particles in the MeV energy range to a sub-micrometer beam spot. The beam optics design has been studied in detail and optimized using detailed ray-tracing simulations and the full mechanical design of the beam line was reported in the Debrecen ICNMTA conference in 2008. During the last two years, the lenses have been carefully aligned and the target chamber has been fully equipped with particle and X-ray detectors, microscopes and precise positioning stages. The beam line is now operational and has been used for its first applications to ion beam analysis. Interestingly, this set-up turned out to be a very versatile tool for a wide range of applications. Indeed, even if it was not intended during the design phase, the ion optics configuration offers the opportunity to work either with a high current microbeam (using the triplet only) or with a lower current beam presenting a sub-micrometer resolution (using the doublet-triplet configuration).

The performances of the CENBG nanobeam line are presented for both configurations. Quantitative data concerning the beam lateral resolutions at different beam currents are provided. Finally, the first results obtained for different types of application are shown, including nuclear reaction analysis at the micrometer scale and the first results on biological samples.
\end{abstract}

\section{Introduction}

During the last decade several research groups around the world have reported the development of focused beam lines presenting a sub-micrometer resolution [1-4]. These nanobeam lines are based either on a single stage of focusing quadrupoles (usually a triplet) or on double stage focusing systems (doublet-doublet, doublet-triplet or triplet-triplet) [5-7]. In comparison with single stage nanobeams, double stage systems present the advantage to achieve higher demagnifications and long working distances.

Such a high resolution nanobeam line, based on a two stage focusing system, has been recently developed on the AIFIRA facility ("Applications Interdisciplinaires des Faisceaux d'Ions en Région Aquitaine") at CENBG (Centre d'Etudes Nucléaires de BordeauxGradignan). A long working distance doublet-triplet configuration was chosen as it presents a good compromise between high demagnification and low spherical aberrations [8,9]. First order calculations allow predicting demagnifications of 65 and 100 in the vertical and horizontal directions, respectively. The AIFIRA nanobeam line is designed for a wide range of applications (chemical analysis at the sub-cellular scale, micro-tomography, nuclear reaction analysis on materials, proton beam writing, etc.) requiring a great flexibility in the adjustment of the beam current, lateral resolution and scanning capabilities. The two stage configuration offers this flexibility as it is possible to easily switch from a triplet configuration for high current analysis to the double stage configuration when a sub-micrometer beam is required. The beam line has been also equipped with an original electrostatic beam scanner allowing to achieve very fast beam motion and complex scanning patterns [10].

This paper presents the beam line performances measured for both configurations (triplet and quintuplet) in terms of beam current and lateral resolution. The beam scanning system, based on a stand-alone sweep controller is also presented. Finally, examples of the first ion beam analysis experiments carried out on the nanobeam line are shown. 


\section{The CENBG nanobeam line}

The CENBG nanobeam line design has been presented in detail previously [8-10]. Briefly, it is installed at the $0^{\circ}$ output of the 3.5 MV Singletron ${ }^{\mathrm{TM}}$ (HVEE, The Netherlands) switching magnet. The beam line is $9.12 \mathrm{~m}$ long and the beam is focused on target using a two stage doublet-triplet configuration of $\mathrm{OM}-50^{\mathrm{TM}}(\mathrm{OX}-$ ford Microbeam Ltd.) magnetic quadrupoles. The object slits are positioned at the waist of the $90^{\circ}$ analyzing magnet $1.2 \mathrm{~m}$ upstream of the switching magnet. An intermediate image is formed by the doublet $2.85 \mathrm{~m}$ upstream of the triplet and the system has a $25 \mathrm{~cm}$ working distance. The beam line is almost entirely shielded with mu-metal to reduce significantly the influence of external stray magnetic fields and is supported by a massive granite monolith to reduce the parasitic vibrations as much as possible. The intermediate image is visualized using a CCD camera by inserting a glass slide covered with a $200 \mathrm{~nm}$ thick $\mathrm{CaF}_{2}$ : $\mathrm{Er}$ scintillating layer and a $10 \times$ objective on the beam axis. An overview of the beam line dimensions can be found on Fig. 1. The beam is scanned over the target using electrostatic scanning plates (maximum scan size: $1.5 \times 1.5 \mathrm{~mm}^{2}$ at $2 \mathrm{MeV}$ ) situated downstream of the triplet and driven by fast high voltage amplifiers (Physicon Corp., Model RS1224-2500). The target chamber is equipped with a full set of motorized stages (Newport ${ }^{\mathrm{TM}}$, maximum range of $100 \mathrm{~mm}$, positioning accuracy of $1 \mu \mathrm{m}$ ) used to position the sample holder, microscope objectives, STIM, RBS (Canberra PIPS detectors, $25 \mathrm{~mm}^{2}$ ) and PIXE (e2v Sirius ${ }^{\mathrm{TM}} 80 \mathrm{~mm}^{2} \mathrm{Si}(\mathrm{Li})$ detector) detectors and faraday cups [10].

\section{Optics alignment}

The achievement of a micrometer or sub-micrometer beam spot at the target position requires a precise alignment of the magnetic quadrupoles. At CENBG, the five magnetic quadrupoles can be moved individually using micrometric screws allowing the adjustment of their position in the transverse plane, rotation and tilt. For alignment purpose, the beam is visualized on $\mathrm{CaF}_{2}$ : $\mathrm{Er}$ scintillating layers at the target position and at the intermediate image position. As the target chamber microscope is equipped with a $20 \times$ objective, a highly magnified image can be used to observe the beam in the focal plane and to finely adjust the quadrupoles positions. As a first step, the triplet has been aligned without the use of the doublet. This procedure is relatively easy as only three lenses, situated in close proximity, are used.

The challenge in aligning a double stage system mainly comes from the fact that the two stages must be aligned together on the same optical axis. In order to achieve this requirement, the triplet was used as a reference. The strategy consisted in first focusing the beam with the triplet and to localize carefully the image position. The quadrupoles forming the doublet were then aligned by looking at the image formed in the target plane (i.e. $3.80 \mathrm{~m}$ downstream of the triplet). The quadrupoles were positioned to obtain an image at the same position as the one obtained with the triplet. This procedure constitutes a first step in the iterative alignment process. The refinement of the optics alignment will be performed as a background task in the near future.

\section{Performances of the beam line}

\subsection{Beam current and lateral resolution}

The beam lateral resolution on target has been measured by scanning the beam on samples presenting a sharp edge. For low current measurements (i.e. STIM), the beam was scanned on a cleaved silicon wafer and the transmitted particles without energy loss were detected with a silicon particle detector situated at $0^{\circ}$. For high current measurements, the beam was scanned on a gold layer deposited on silicon and eroded using a FIB (Focused lon Beam). For the resolution measurements, particles backscattered on the gold atoms were detected. Using both methods, beam profiles were obtained (see an example on Fig. 2) and fitted by a Gauss error function using the ROOT analysis software [11]. Results obtained for both focusing configurations are presented in Table 1.

The triplet configuration allows the focusing of the beam in a sub-micron spot for low current applications (i.e. STIM imaging). Interestingly, using this configuration, a rather high current on target $(>300 \mathrm{pA})$ can be obtained together with a $1 \mu \mathrm{m}$ beam size. Depending on the beam parameters and the condition of the ion source, beam currents up to $600 \mathrm{pA}$ on target have been measured. The use of this configuration is thus suitable for PIXE/RBS analysis of thin targets (i.e. cell monolayers) for which a high beam current is needed to keep reasonable acquisition times.

The quintuplet configuration turned out to be more difficult to use in a routine way. It allows to obtain beam sizes of about $200 \mathrm{~nm}$ for STIM analysis. This resolution is better than the one measured using the triplet configuration but is above the one expected from the ray-tracing simulations $(<100 \mathrm{~nm}$ when a $5 \mu \mathrm{m}$ object aperture is used). High current measurements show less reproducible results. It was possible to obtain a demagnification of about 90 in one plane and 62 in the other plane. Indeed, a beam size of $1.1 \times 1.6 \mu \mathrm{m}$ was measured when using a $100 \mu \mathrm{m}$ circular object. These values are close to the theoretical one (100 and 65 respectively) but the image size did not decrease with the object size in a linear way, indicating a strong effect of the parasitic aberrations. Indeed, a small misalignment of the doublet can lead to large aberrations since it is situated several meters away from the triplet. Additionally, rather low currents on target were measured compared to the triplet configuration (about $50 \mathrm{pA}$ with a $100 \mu \mathrm{m}$ object). This decrease can be due to several factors. Indeed, a mechanical misalignment of the doublet can slightly steer the beam out of the optical axis of the triplet and thus a lower current

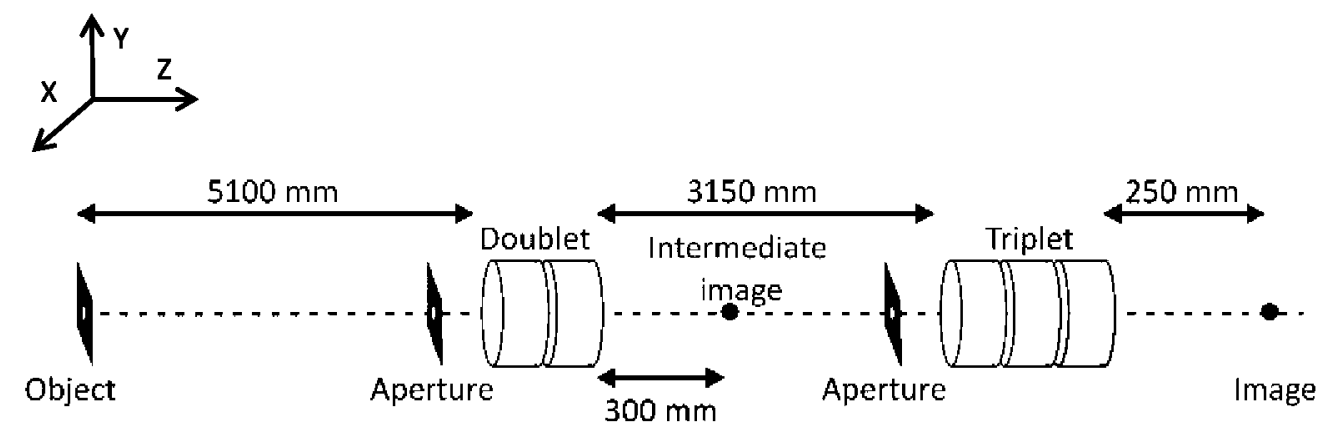

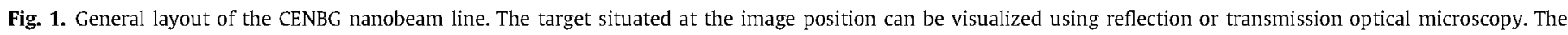
intermediate image is visualized using a microscope. 

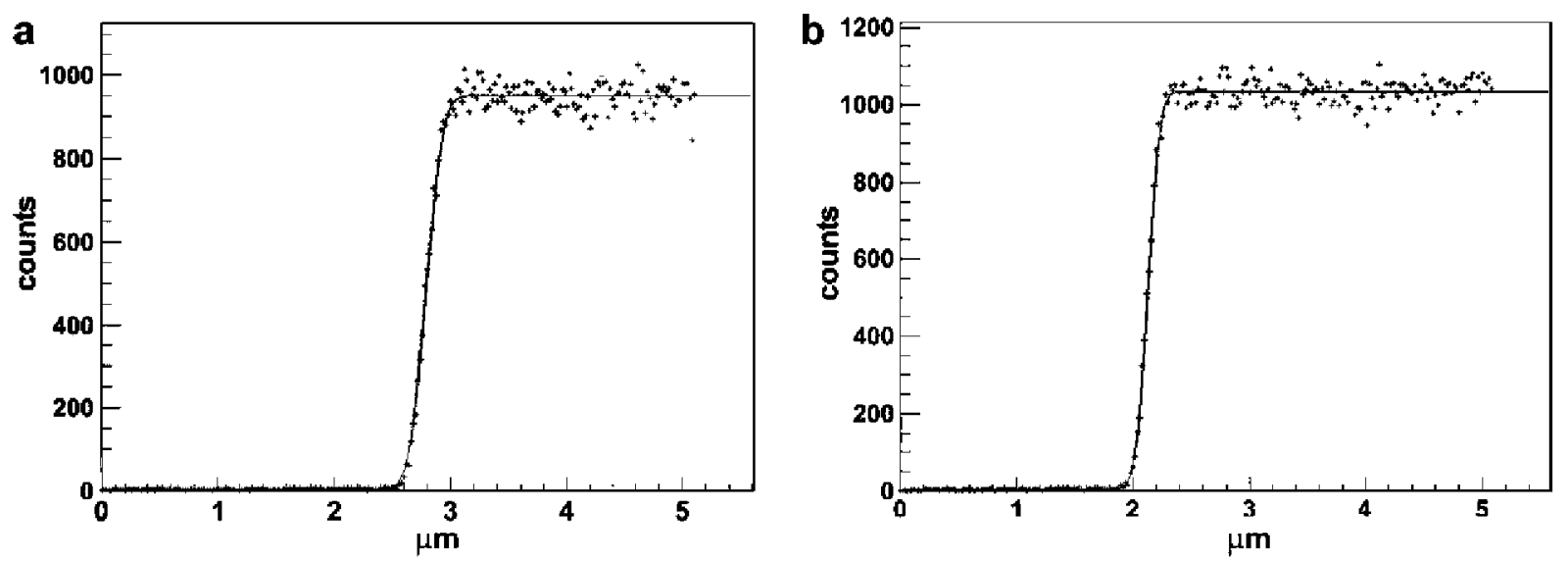

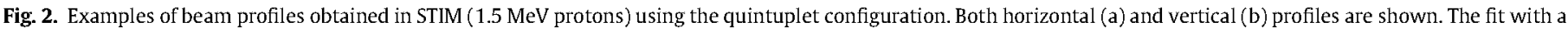

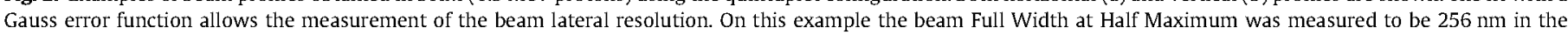
horizontal direction (a) and $187 \mathrm{~nm}$ in the vertical direction (b).

Table 1

Summary of the beam lateral resolution on target as a function of the beam current for both focusing configurations: triplet and quintuplet. Numerical values are indicative values obtained on several independent experiments.

\begin{tabular}{llllll}
\hline & \multicolumn{2}{l}{ Low current mode } & & \multicolumn{2}{l}{ High current mode } \\
\cline { 2 - 3 } & Beam current (ions/s) & Lateral resolution $(\mathrm{nm})$ & & Beam current $(\mathrm{pA})$ & Lateral resolution $(\mu \mathrm{m})$ \\
\hline Triplet & 2000 & $300 \times 300$ & 300 & 1 \\
Quintuplet & 700 & $200 \times 250$ & 30 & 1.5 \\
\hline
\end{tabular}

passes through the aperture located before the triplet. In addition, the doublet introduces a beam divergence after the intermediate image, leading to a smaller beam brightness at the position of the aperture. These effects will have to be investigated in more details and more efforts will have to be put on the quadrupole alignment in order to enhance the resolution of the nanobeam.

\subsection{Beam scanning and control}

The development of a multi-purpose nanobeam line requires the use of a very flexible beam control system. For this reason, it was chosen to develop a stand-alone sweep controller based on a compact Reconfigurable Input/Output (compact RIO) device from National Instrument ${ }^{\mathrm{TM}}$. This system is loaded with a dedicated control software written in LabVIEW ${ }^{\mathrm{TM}}$ and can be addressed by any computer of the experimental hall via a web browser or any home-made program supporting TCP/IP messages. The scanning voltages are sent simultaneously on the analog outputs $( \pm 10 \mathrm{~V}$, 16 bits DAC) for beam deflection and on the digital outputs for real-time data acquisition. The developed system allows to produce maps with a $256 \times 256$ pixels resolution and at a relatively high scanning speed as it is possible to go down to $15 \mu$ s per pixel (limited by the DAC conversion time). The compact RIO is also equipped with high speed TTL pulse counters ( $>2 \mathrm{MHz}$ ), for builtin high count rate imaging and particle counting, and with high speed TTL outputs used to generate beam shutter or deadtime signals. Arbitrary scanning patterns with more than 130,000 positions can be defined. This makes it particularly useful for imaging of complex structures, ion beam lithography and targeted irradiation.

\section{First use for ion beam analysis}

\subsection{STIM and PIXE analysis of biological samples}

One of the major applications of the CENBG nanobeam line is the analysis of biological samples at a sub-cellular scale. This appli- cation, initially developed on the former microbeam facility, is recognized as a powerful tool for the quantification and localization of inorganic elements in cells [12]. Using the CENBG nanobeam line, it is possible to combine STIM imaging at high resolution (about $200 \mathrm{~nm}$ ) to visualize the cell density distribution and PIXE analysis with a resolution of about $1 \mu \mathrm{m}$ to map and quantify inorganic elements. At this beam resolution, it is possible to clearly localize subcellular compartments (nucleus, cytoplasm). As an example, Fig. 3 shows STIM and PIXE maps obtained on human primary keratinocytes expose to $\mathrm{TiO}_{2}$ nanoparticles. Thanks to the high resolution of the beam, $\mathrm{TiO}_{2}$ nanoparticles aggregates located in close vicinity of the cell nucleus appear on STIM maps. This observation is confirmed by PIXE maps where the X-ray signal from titanium is clearly co-localized with the dense spots observed on the STIM map. A complete study has been recently performed at CENBG on the former microbeam line [13]. The nanobeam line opens new opportunities in mapping biological cells at the submicrometer scale in particular in the area of nanotoxicology research.

\subsection{Nuclear reaction analysis at the micrometer scale}

The full remote control of the nanobeam line allows the use of high currents deuteron beams for nuclear reaction analysis (NRA) at the micrometer scale without radiation hazard for the experimenter. The beamline was recently used to map the nitrogen content in Zircaloy fuel cladding tubes. Indeed, in the area of nuclear plant accidental scenario analysis, understanding of the oxidation mechanisms of Zircaloy fuel cladding tubes when exposed to air at high temperature is of particular importance. Specifically, the exact role of nitrogen in the reaction path needs to be clarified. The ${ }^{14} \mathrm{~N}(\mathrm{~d}, \mathrm{p}){ }^{15} \mathrm{~N}$ reaction was used to measure the nitrogen distribution in zirconia layers formed on Zircaloy-4 alloy samples exposed to air at temperatures between 800 and $1000^{\circ} \mathrm{C}$. NRA was performed with a $1.8 \mathrm{MeV}$ deuteron beam. The size of the beam has been estimated to be $4 \mu \mathrm{m}$ and the current on target was close to $2 \mathrm{nA}$. The particles resulting from the nuclear reactions $\left(\mathrm{H}^{+}\right.$, 

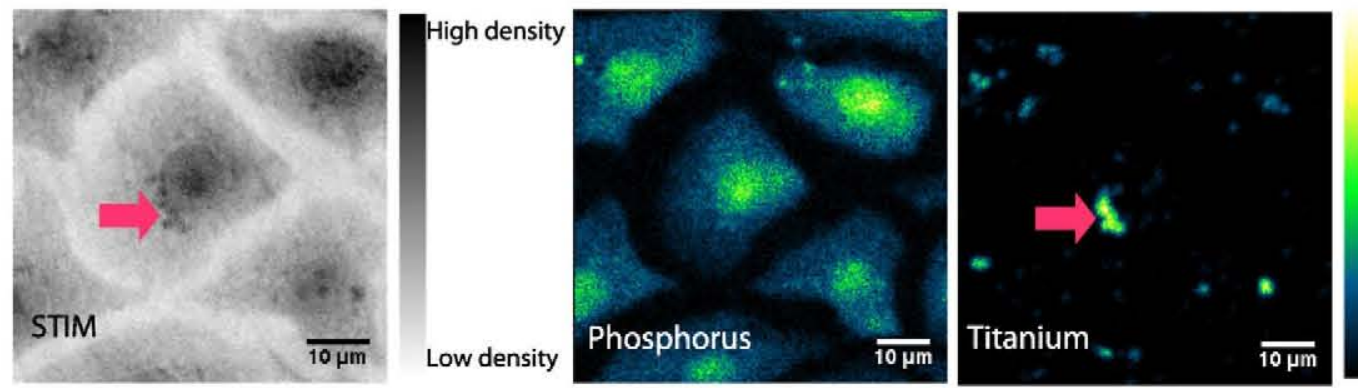

Xray counts
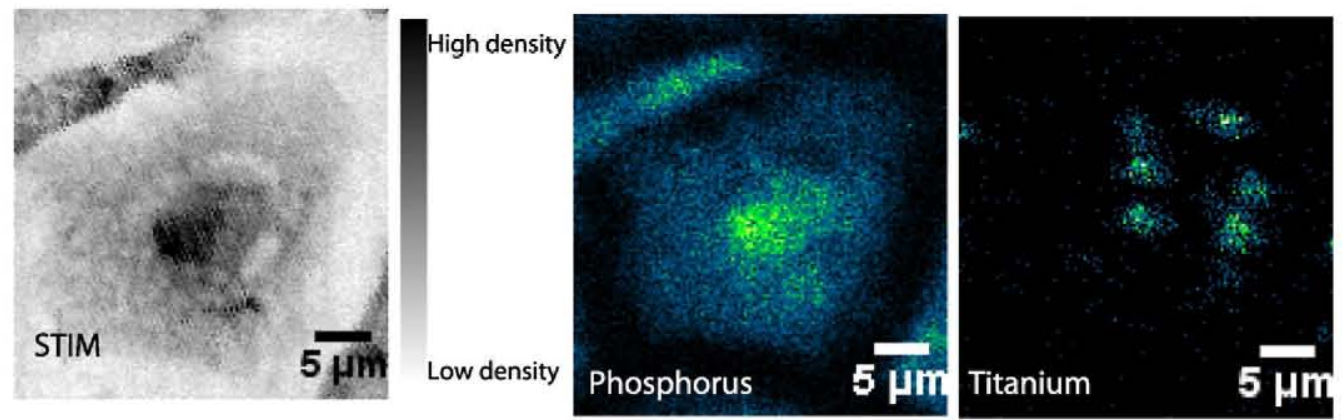

Xray counts

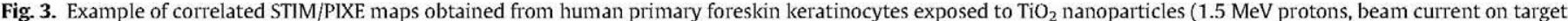

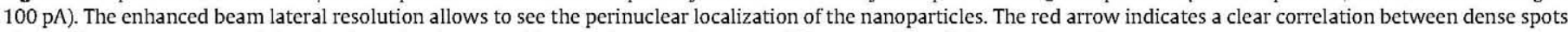
observed on STIM maps and the titanium signal. Top and bottom images show two different regions of the same sample.
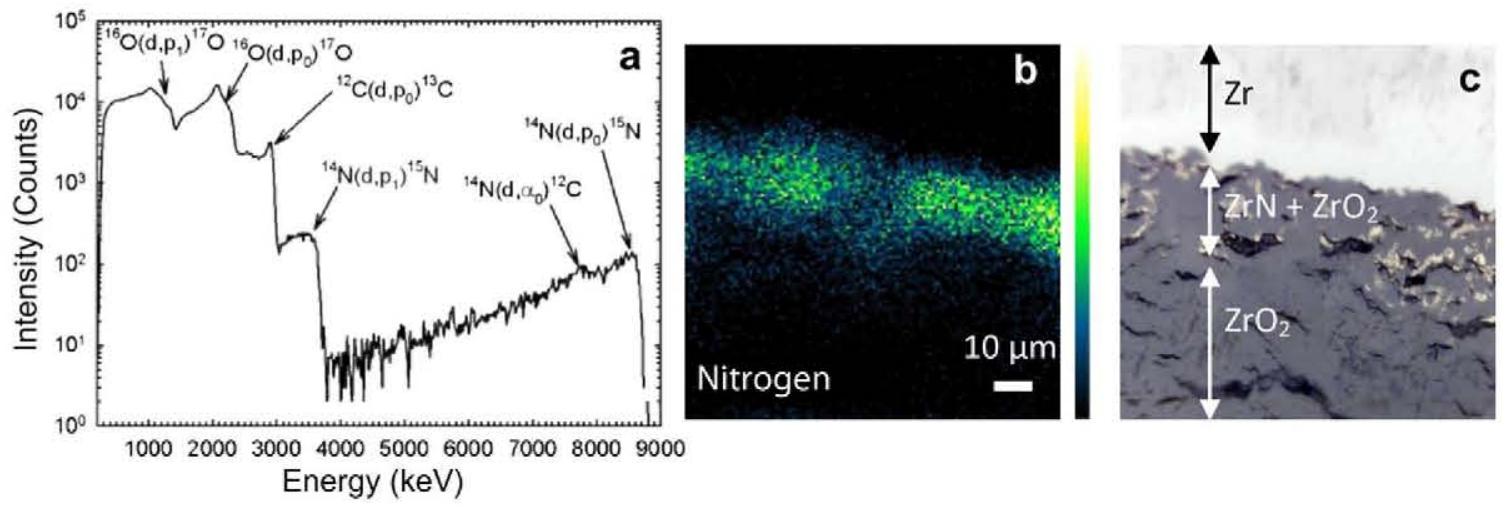

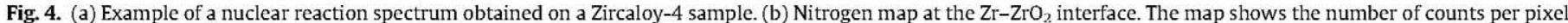
corresponding to the ${ }^{14} \mathrm{~N}\left(\mathrm{~d}, \mathrm{p}_{0}\right)^{15} \mathrm{~N}$ reaction. (c) Corresponding optical image.

$\mathrm{He}^{++}$) were collected with a Silicon detector (Canberra PIPS detector, $25 \mathrm{~mm}^{2}$ ) at $135^{\circ}$. An X-Rays detector was also used to monitor the $\mathrm{Zr}$ signal. Both detectors were protected with Mylar foil of 37 and $13 \mu \mathrm{m}$, respectively. Fig. 4 shows the nitrogen distribution for a zirconia layer formed at $950{ }^{\circ} \mathrm{C}$ in air. No nitrogen was detected (detection limit: $100 \mathrm{ppm}$ ) in the metallic part of the sample; nitrogen is mainly detected in the $\mathrm{ZrO}_{2}-\mathrm{ZrN}$ mixed region and $\mathrm{ZrN}$ particles are mainly located at the metal/oxide interface. This first experiment shows the ability of the CENBG nanobeam line to perform NRA at the micrometer scale.

\section{Concluding remarks}

The CENBG nanobeam line is now operational to perform ion beam analysis at the micrometer scale and a sub-micrometer beam spot can be obtained in the case of low fluence analysis (STIM). This beam line is a very versatile tool allowing the use of a wide range of ion beam analysis techniques. The optimal lateral resolution, predicted using detailed simulations, has not yet been obtained experimentally. To achieve this goal, the beam optics alignment will have to be optimized and more precise beam diagnostic systems will have to be developed. Nevertheless, beam resolutions below $300 \mathrm{~nm}$ in low current mode and of about $1 \mu \mathrm{m}$ in high current mode can be achieved in a routine way and open new opportunities to perform ion beam analysis and irradiation at a sub-micrometer scale.

\section{Acknowledgements}

The authors would like to thank F. Delalée, L. Serani and P. Alfaurt for their support in the technical developments on the beamline, R. Ortega, G. Devès, A. Carmona and S. Roudeau for fruitful discussions about the analysis of biological samples and $C$. Duriez and S. Guilbert from IRSN Cadarache for their work on the NRA analysis. 
This work is partially supported by the European Community as an Integrating Activity "Support of Public and Industrial Research Using Ion Beam Technology (SPIRIT)" under EC Contract No. 227012 .

\section{References}

[1] F. Watt, J.A. Van Kan, I. Rajta, A.A. Bettiol, T.F. Choo, M.B.H. Breese, T. Osipowicz The National University of Singapore high energy ion nano-probe facility: Performance tests, Nucl. Instr. and Meth. B 210 (2003) 14

[2] S. Matsuyama, K. Ishii, H. Yamazaki, Y. Kikuchi, K. Inomata, Y. Watanabe, A Ishizaki, R. Oyama, Y. Kawamura, T. Yamaguchi, G. Momose, M. Nagakura, M. Takahashi, T. Kamiya, Progress and application of the Tohoku microbeam system, Nucl. Instr. and Meth. B 260 (2007) 55.

[3] Tilo Reinert, Daniel Spemann, Markus Morawski, Thomas Arendt, Quantitative trace element analysis with sub-micron lateral resolution, Nucl. Instr. and Meth. B 249 (2006) 734

[4] G. Dollinger, G. Datzmann, A. Hauptner, R. Hertenberger, H.J. Körner, P. Reichart, B. Volckaerts, The Munich ion microprobe: Characteristics and prospect, Nucl. Instr. and Meth. B 210 (2003) 6.

[5] M. Rothermel, T. Butz, T. Reinert, Rearranging a nanoprobe: Line foci, grid shadow patterns and performance tests, Nucl. Instr. and Meth. B 267 (2009) 2017
[6] M.J. Merchant, G.W. Grime, K.J. Kirkby, R. Webb, A survey of two-stage focusing systems for nanobeam design, Nucl. Instr. and Meth. B 260 (2007) 8.

[7] G.A. Glass, A.D. Dymnikov, B. Rout, D.P. Zachr, High energy focused ion beam technology and applications at the Louisiana Accelerator Center, Nucl. Instr. and Meth. B 260 (2007) 372

[8] S. Incerti, Q. Zhang, F. Andersson, Ph. Moretto, G.W. Grime, M.J. Merchant, D.T. Nguyen, C. Habchi, T. Pouthier, H. Seznec, Monte Carlo simulation of the CENBG microbeam and nanobeam lines with the Geant4 toolkit, Nucl. Instr. and Meth. B 260 (2007) 20.

[9] F. Andersson, Ph. Barberet, S. Incerti, Ph. Moretto, A detailed ray-tracing simulation of the high resolution microbeam at the AIFIRA facility, Nucl. Instr. and Meth. B 266 (2008) 1653.

[10] Ph. Barberet, S. Incerti, F. Andersson, F. Delalee, L. Serani, Ph. Moretto, Technical description of the CENBG nanobeam line, Nucl. Instr. and Meth. B 267 (2009) 2003.

[11] ROOT web site, Available from: <http://www.root.cern.ch>

[12] A. Carmona, G. Devès, R. Ortega, Quantitative micro-analysis of metal ions in subcellular compartments of cultured dopaminergic cells by combination of three ion beam techniques, Anal. Bioanal. Chem. 390 (2008) 1585.

[13] M. Simon, P. Barberet, M.H. Delville, P. Moretto and H. Seznec, Titanium dioxide nanoparticles induced intracellular calcium homeostasis modification in primary human keratinocytes. Towards an in vitro explanation of titanium dioxide nanoparticles toxicity, Nanotoxicology, in press. 